\title{
Quantifying Memory Capacity as a Quantum Thermodynamic Resource
}

\author{
Varun Narasimhachar, ${ }^{1, *}$ Jayne Thompson, ${ }^{2}$ Jiajun Ma, ${ }^{3}$ Gilad Gour, ${ }^{4}$ and Mile Gu ${ }^{1,2, \dagger}$ \\ ${ }^{1}$ Complexity Institute and School of Physical and Mathematical Sciences, Nanyang Technological University, \\ 50 Nanyang Ave, 639798, Singapore \\ ${ }^{2}$ Centre for Quantum Technologies, National University of Singapore, Block S15, 3 Science Drive 2, 117543, Singapore \\ ${ }^{3}$ Center for Quantum Information, Institute for Interdisciplinary Information Sciences, \\ Tsinghua University, 100084 Beijing, China \\ ${ }^{4}$ Institute for Quantum Science and Technology and Department of Mathematics and Statistics, \\ University of Calgary, 2500 University Drive NW, Calgary, T2N 1N4 Alberta, Canada
}

(Received 21 May 2018; revised manuscript received 16 September 2018; published 13 February 2019)

\begin{abstract}
The information-carrying capacity of a memory is known to be a thermodynamic resource facilitating the conversion of heat to work. Szilard's engine explicates this connection through a toy example involving an energy-degenerate two-state memory. We devise a formalism to quantify the thermodynamic value of memory in general quantum systems with nontrivial energy landscapes. Calling this the thermal information capacity, we show that it converges to the nonequilibrium Helmholtz free energy in the thermodynamic limit. We compute the capacity exactly for a general two-state (qubit) memory away from the thermodynamic limit, and find it to be distinct from known free energies. We outline an explicit memory-bath coupling that can approximate the optimal qubit thermal information capacity arbitrarily well.
\end{abstract}

DOI: $10.1103 /$ PhysRevLett.122.060601

Szilard's adaptation of the Maxwell's demon thought experiment, supplemented by Landauer's principle, illustrates a compelling connection between an entity's capacity to store information, on the one hand, and its capacity to deliver thermodynamic work on the other [1-4]. In particular, the Szilard engine relies on storing information in an energy-degenerate two-state memory system. If such a memory is initialized in some pure state, one bit of information can be recorded onto it without expending any free energy. At the other extreme, if the memory started out in a maximally mixed state, no further information could be encoded onto it without first erasing its contents, which would entail tapping into an external free energy source.

This simple special case exemplifies a deeper connection between a memory's information capacity and its athermality (i.e., departure from thermal equilibrium). How does this connection manifest in a general scenario where the memory is quantum mechanical, with internal states of differing energetic values? The athermality of such a memory may involve coherent superposition of energy eigenstates [5-14]. In addition to the classical laws of thermodynamics, more general principles of nonequilibrium quantum thermodynamics [15-26] would then apply.

Published by the American Physical Society under the terms of the Creative Commons Attribution 4.0 International license. Further distribution of this work must maintain attribution to the author(s) and the published article's title, journal citation, and DOI.
In this Letter we formalize the thermodynamic value of memory capacity for quantum systems with general energy landscapes. To this end, we conceptualize a thermally passive memory: writing onto such a memory is constrained to use no thermodynamic resource other than what the memory's initial state carries intrinsically. We define the resulting capacity as the given state's thermal information capacity. We show that in the thermodynamic limit, this measure recovers the standard nonequilibrium free energy. We also compute the thermal information capacity exactly for the case of a single two-level ("qubit") system away from the thermodynamic limit, establishing it as a distinct measure of athermality with operational relevance. We discuss a potential practical scheme to write onto a single-qubit memory at a rate arbitrarily close to the capacity, finding a tradeoff between implementation speed and closeness of approximation.

Framework.-We aim to capture the precise relationship between the thermodynamic inequilibrium ("athermality") in arbitrary quantum states $\rho$ of a memory, and its storage capacity. To this end, we envision a thermally passive memory (Fig. 1): a quantum system $\mathbf{M}$, initialized in state $\rho$, in a thermal environment of uniform temperature $T$. This effectively cuts off $\mathbf{M}$ from any external sources of free energy, rendering it thermally passive.

Consider an arbitrary classical random variable $K \equiv\left\{\left(p_{k}, k\right)\right\}$, representing information to be recorded onto M. This entails applying some $k$-dependent operation on $\mathbf{M}$ that transforms $\rho$ to a corresponding "codeword" state $\sigma^{(k)}$. Now, if $\rho$ were the state of thermal equilibrium, it 


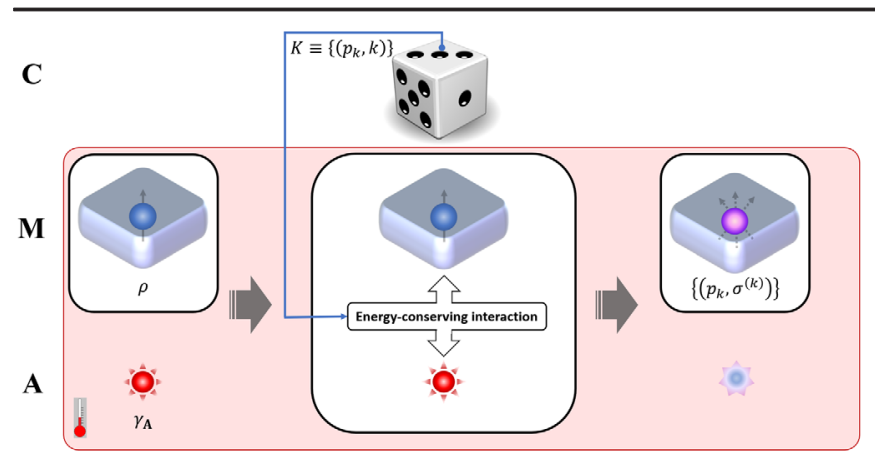

FIG. 1. Thermally passive memory: the pertinent information variable $K \equiv\left\{\left(p_{k}, k\right)\right\}$ (contained in a classical system $\mathbf{C}$ ) is recorded on the quantum memory $\mathbf{M}$ through a $k$-dependent energy-conserving interaction of $\mathbf{M}$ with an auxiliary system $\mathbf{A}$ in its thermal state $\gamma_{\mathbf{A}}$, transforming the memory's initial ("blank tape") state $\rho$ to the ensemble $\left\{\left(p_{k}, \sigma^{(k)}\right)\right\}$ of quantum statevalued codewords. No free energy is used in this process, except that already present in $\rho$.

would be impossible to take $\mathbf{M}$ to any other state passively, making it useless as a memory. Any capacity for $\mathbf{M}$ to passively record information, therefore, owes to the athermality of $\rho$.

Thermally passive encoding is formally captured by thermal operations [20], which describe the possible state transformations of a system in contact with a single thermal bath. Left to equilibrate with the environment, M would eventually reach its thermal, or Gibbs, state $\gamma \propto \exp \left(-H_{\mathbf{M}} / k_{B} T\right)$, where $H_{\mathbf{M}}$ is its free Hamiltonian. In this process of thermalization, $\mathbf{M}$ loses free energy and all other aspects of thermodynamic resourcefulness. A thermal operation is a more general type of resource-depleting process, of which thermalization is a special case. It is an interaction of $\mathbf{M}$ with a thermal auxiliary system $\mathbf{A}$ (Fig. 1): It starts with $\mathbf{M}$ in some initial state $\rho$ uncorrelated with $\mathbf{A}$ (which, by virtue of being thermal, is in its own local Gibbs state $\gamma_{\mathrm{A}}$ ), followed by turning on an arbitrary energyconserving interaction between $\mathbf{M}$ and $\mathbf{A}$, and then decoupling the two again. The choice of system $\mathbf{A}$ is left arbitrary, so long as it is prepared in the Gibbs state determined by its own Hamiltonian and the bath's temperature.

Passively encoding the classical variable $K$ on $\mathbf{M}$ effectively transforms its state from $\rho$ to an ensemble $\mathcal{C} \equiv\left\{\left(p_{k}, \sigma^{(k)}\right)\right\}$, where $\sigma^{(k)}=\mathcal{T}^{(k)}(\rho)$ with $\mathcal{T}^{(k)}$ some thermal operation for every $k$. The maximum amount of information that can be reliably recovered from $\mathcal{C}$ by unrestricted readout is then given by its Holevo information:

$$
\chi(\mathcal{C})=S\left(\sum_{k} p_{k} \sigma^{(k)}\right)-\sum_{k} p_{k} S\left(\sigma^{(k)}\right)
$$

where $S(\cdot)$ denotes the von Neumann entropy. For a given initial state $\rho$, define $\mathscr{C}(\rho)$ as the set of all codes $\mathcal{C}$ consisting of codewords $\sigma^{(k)}$ accessible from $\rho$ by thermal operations [27]. This set represents all possible ways that classical information can be written passively onto $\mathbf{M}$, allowing arbitrary variations in the classical variable $K$ being written. Our main quantity of interest is the optimal amount of information that can be written in this way, given an initial resource state.

Definition 1.-(Thermal information capacity) The thermal information capacity (TIC) of the thermal memory $\mathbf{M}$ initialized in blank state $\rho$ is defined as

$$
I_{\text {th }}(\rho):=\sup _{\mathcal{C} \in \mathscr{C}(\rho)} \chi[\mathcal{C}]
$$

From the properties of the Holevo information, it follows that the TIC is always non-negative. Another property of the TIC is that, as a function of the input state, it is strictly nonincreasing under thermal operations:

$I_{\text {th }}(\mathcal{T}[\rho]) \leq I_{\text {th }}(\rho) \quad \forall \rho, \quad \forall$ thermal operations $\mathcal{T}$.

Thus, as expected, the TIC is a measure of thermodynamic resourcefulness of the state $\rho$, akin to free energy functions: a thermal operation acting on a given state can only result in a state with equal or lower TIC. It vanishes only when $\rho=\gamma$, and is positive otherwise.

Note that $I_{\text {th }}$ is the absolute maximum amount of information that we can encode within $\mathbf{M}$, in a single shot, without energy expenditure. In particular, we assume no restriction on the operations required to decode $K$ from $\sigma^{(k)}$, either to single-shot processing or by energy considerations. Our primary motivation here is foundational: this allows us to study the efficacy of the writing process considered in isolation (e.g., the first step in a Szilard engine), and relate it to the initial athermality in $\mathbf{M}$. Nevertheless, $I_{\text {th }}$ has direct operational relevance in the context of remote probes operating in energy-depleted environments. Such probes are constrained in their ability to harness free energy to store the information in their environment. The readout of this information may not need to be executed immediately, and may instead be deferred for more favorable conditions (e.g., after the probe has returned to a powered central facility). Examples of such settings arise in quantum sensing, where the operations that encode environmental data are generally thermal (e.g., unitary Hamiltonian evolution in metrology [29,30], or beam splitter interactions with a thermal environment in the case of quantum illumination [31-33]).

Thermodynamic limit. - A helpful point to start investigating the TIC is to consider its thermodynamic limit, which concerns the average behavior over a large number of independent, identically prepared (i.i.d.) instances. What is the optimal TIC per copy of a resource state $\rho$, in the thermodynamic limit? More precisely, this quantity is defined as 


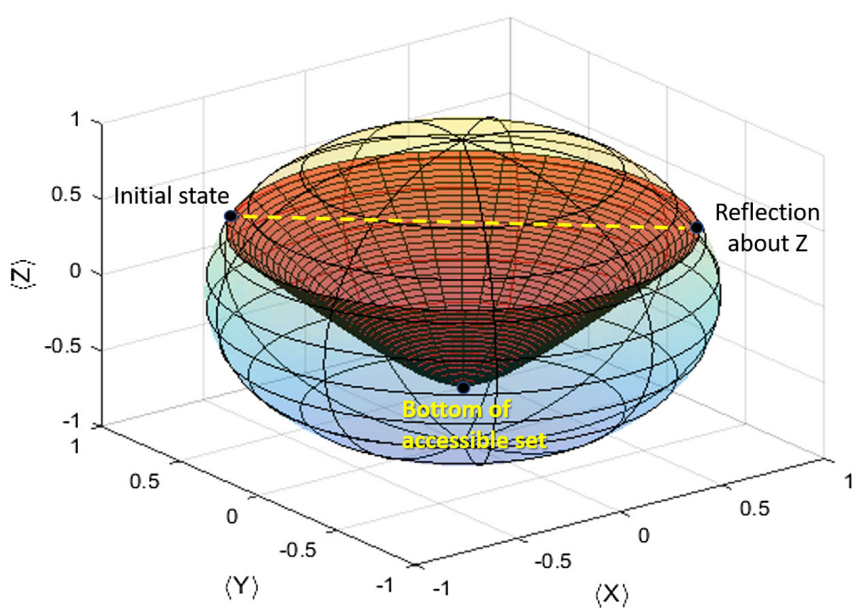

FIG. 2. Bloch visualization of the set of states accessible by qubit thermal operations from a pure initial state; an informationally maximal code constructed from the accessible set comprises the three indicated extremal states as codewords.

$$
I_{\mathrm{th}}^{\infty}(\rho):=\lim _{m \rightarrow \infty} \frac{I_{\mathrm{th}}\left(\rho^{\otimes m}\right)}{m} .
$$

Apart from its own operational significance, the limiting i.i.d. value is useful as an upper bound on the single-copy TIC. While the latter is in general difficult to compute, the i.i.d. limit can be calculated exactly using the theory of asymptotic equipartition, leading to the following result.

Proposition 1.-In the thermodynamic limit of infinitely many, independent and identically distributed (i.i.d.) copies, the optimal thermal information capacity per copy of a memory state $\rho$ is given by $I_{\mathrm{th}}^{\infty}=F(\rho)$, a quantum nonequilibrium generalization of the Helmholtz free energy [34], defined as the quantum relative entropy of $\rho$ with respect to the Gibbs state $\gamma$ :

$$
F(\rho):=S(\rho \| \gamma) \equiv \operatorname{Tr}\left(\rho \log _{2} \rho\right)-\operatorname{Tr}\left(\rho \log _{2} \gamma\right)
$$

We provide the proof in Supplemental Material S1. Notably, the free energy emerges as the asymptotic TIC despite the readout's being unrestricted. This is because the optimal asymptotic code consists of pure eigenstates all equal in energy, and can therefore be read out by an energyconserving measurement. Our finding establishes that the TIC recovers standard notions of free energy in the thermodynamic limit $[24,35]$.

We now turn to the study of the TIC in the non-i.i.d., or single-shot, regime. The science of general coherent thermal operations in this regime is nontrivial, but the special case of two-level systems, or qubits, is relatively tractable.

2-level memory.-Consider a qubit memory $\mathbf{M}$ governed by a (generally nondegenerate) Hamiltonian $H_{\mathbf{M}}=$ $E_{0}|0\rangle\left\langle 0\left|+E_{1}\right| 1\right\rangle\langle 1|$ and immersed in an ambient temperature $T$. Computing the TIC (Definition 1) of a given initial
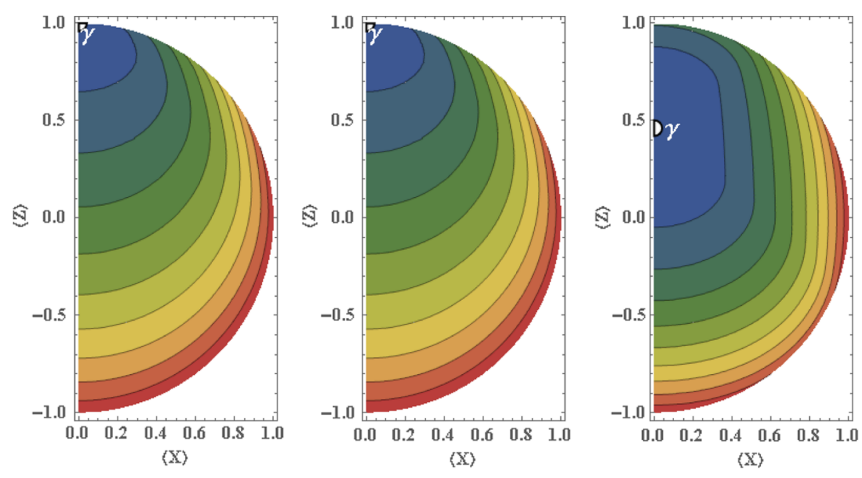

(a) $T=0$

(b) $T=0.1 \Delta E / k_{\mathrm{B}}$

(c) $T=\Delta E / k_{\mathrm{B}}$
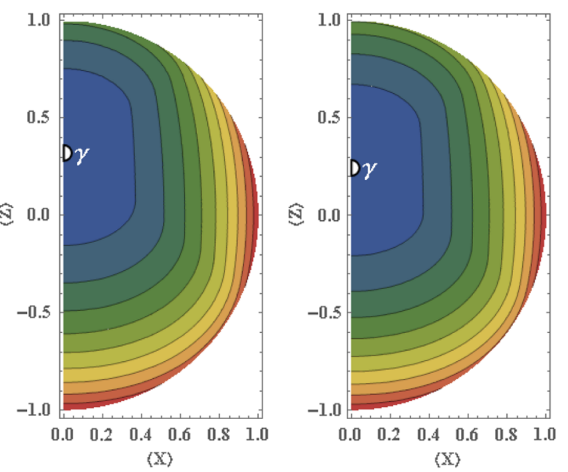

(e) $T=2 \Delta E / k_{\mathrm{B}}$

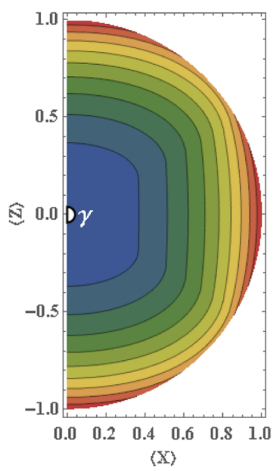

(f) $T \rightarrow \infty$

(d) $T=1.5 \Delta E / k_{\mathrm{B}}$

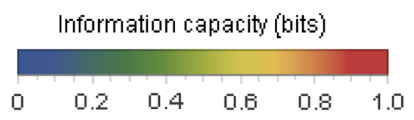

FIG. 3. Thermal information capacity (TIC) over different blank-memory states in the $X^{+} Z$ section of the Bloch ball, for a qubit memory (with energy gap $\Delta E$ ) at various temperatures. The TIC of the Gibbs state $\gamma$ is zero, and is higher for states further away from $\gamma$. The zero-temperature limit behaviour persists at temperatures as high as $0.1 \Delta E / k_{\mathrm{B}}$; significant variation ensues in the $\mathcal{O}\left(\Delta E / k_{\mathrm{B}}\right)$ temperature range, while the hightemperature limit resembles the information landscape of a non-energy-degenerate qubit memory.

state $\rho$ entails searching from the set $\mathscr{C}(\rho)$ of codes accessible from $\rho$. The concavity of the von Neumann entropy function implies that codes containing only extreme points of the accessible set will attain the optimum. This and other simplifications (detailed in Supplemental Material S2 [36]) lead to our main result:

Theorem 1.-For a qubit memory $\mathbf{M}$, an optimal code accessible thermally from an initial state $\rho$ is of the form

$$
\mathcal{C}_{q} \equiv\left\{\left(\frac{q}{2}, \rho\right),\left(\frac{q}{2}, Z \rho Z\right),(1-q, \tilde{\rho})\right\}
$$

where $q \in[0,1], Z=|0\rangle\langle 0|-| 1\rangle\langle 1|$, and $\tilde{\rho}$ is the state at the tip of the accessible set (Fig. 2). The thermal information capacity (TIC) of $\rho$ can then be determined by carrying out the single-parameter optimization $I_{\text {th }}(\rho)=\max _{q \in[0,1]} \chi\left(\mathcal{C}_{q}\right)$. 


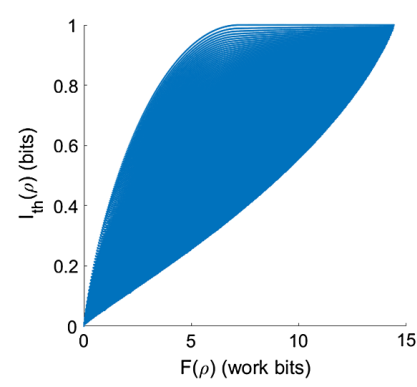

(a) $T=0.1 \Delta E / k_{\mathrm{B}}$

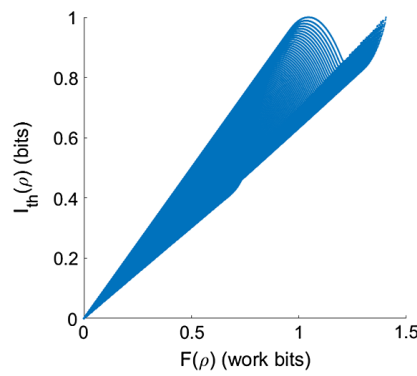

(c) $T=2 \Delta E / k_{\mathrm{B}}$

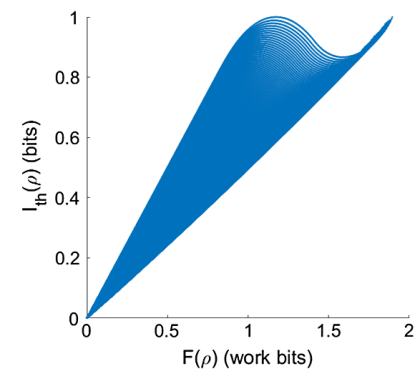

(b) $T=\Delta E / k_{\mathrm{B}}$

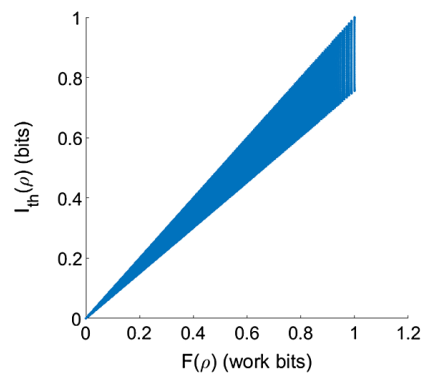

(d) $T \rightarrow \infty$

FIG. 4. Scatter plots of the thermal information capacity vs nonequilibrium Helmholtz free energy of qubit memory states: while the two resources are correlated in their state dependence, they are distinct, particularly at lower temperatures. In each plot, the top-right point of maximum capacity corresponds to the initial state $\rho=|1\rangle\langle 1|$, the pure excited state. The maxima occurring to the left of this point correspond to initial states along the equator, e.g., $\rho=|+\rangle\langle+|$. In the $T \rightarrow \infty$ limit, the two maximal regions get more and more similar in their free energy, as the latter converges to the purity (or "negentropy") of $\rho$.

This optimization can be easily carried out numerically. Figure 3 depicts the result: the TIC as a function of the initial state $\rho$, at various temperatures measured in relation to $\Delta E \equiv E_{1}-E_{0}$. The TIC understandably vanishes when $\rho$ equals the Gibbs state $\gamma$, and increases with athermality, i.e., the departure of $\rho$ from this state. The Helmholtz free energy $F(\rho)$ [Eq. (5)] is an operationally meaningful measure of athermality, and so we investigate the behavior of $I_{\text {th }}(\rho)$ in relation to $F(\rho)$ (Fig. 4). We see that the two resources vary similarly with $\rho$, but less so at lower temperatures. In Supplemental Material S3 [36], we examine the TIC in relation with other resourcefulness measures, namely, the purity and the relative entropy of coherence; we find the free energy to be better than these other resources as an indicator of the TIC. This is understandable, given the asymptotic convergence of the TIC to the free energy (Proposition 1).

Towards implementation.-The thermal operations framework, which we have used to model the encoding process, is agnostic about the existence of a practically feasible auxiliary system $\mathbf{A}$ and coupling to realize a desired thermal operation (see Ref. [37] for a detailed discussion). Thus, we would like to go beyond the abstraction of thermal operations and construct a concrete

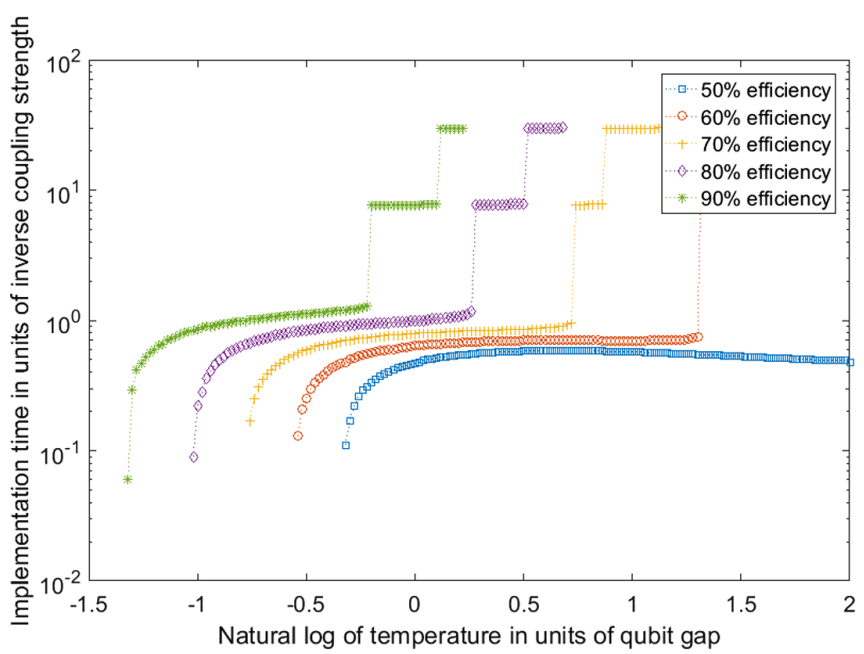

FIG. 5. Time taken by a Jaynes-Cummings coupling to approximate the optimal qubit thermal information capacity to various efficiencies, vs bath temperature. The speed-efficiency tradeoff is reminiscent of a heat engine's performance.

realization. To this end, we now probe an interaction of the qubit memory $\mathbf{M}$ with a bosonic mode bath tuned to M's energy gap, interacting with the latter via a JaynesCummings coupling.

We refer again to Fig. 2 showing the three states constituting an optimal code obtainable from a given initial state. The initial state itself being one of these, another results from reflecting the initial state about the Pauli $Z$ axis, while the third lies at the tip of the convex cone of accessible states. Reflection about $Z$ is represented by the unitary transformation $Z$, which can be effected simply by evolving the memory system under its free Hamiltonian for a suitable length of time. Transforming to the third codeword state, however, requires population inversion relative to the initial state, which cannot be achieved perfectly by a Jaynes-Cummings coupling owing to asynchronicity between the Rabi oscillations within different memorybath energy levels. Nevertheless, we found that the optimal capacity can be approximated arbitrarily well, albeit at the cost of longer running time (Fig. 5): this mirrors the powerefficiency tradeoff in the performance of heat engines. The phase transitionlike jumps occur due to the abovementioned Rabi oscillations whose collective effect on the qubit's marginal state is irregular in time. The degree of population inversion required to meet a given efficiency is generally achieved at similar times over short ranges of temperature, but at certain critical temperatures where it just begins to fail, the irregular time dependence of the population inversion leads to a long period of oscillations where this failure persists, until a sufficient inversion level is finally reached around a different time regime. This new inversion level again remains sufficient to meet the required efficiency, until the next critical temperature is hit, and so on. The downward dip of some of the curves with increasing temperature seems counterintuitive. We 
conjecture that this is a consequence of the fall in optimal capacity with increasing temperature, thus rendering it easier to approach. Technical details about these results are provided in the Supplemental Material S4 [36].

Discussion. - We probed the thermodynamical limitations of the capacity of a quantum system to store information. We defined a thermally passive quantum memory as one which is written onto without access to free energy sources, and thermal information capacity as the capacity of such a memory. After determining that the thermal information capacity approaches the nonequilibrium free energy in the thermodynamic limit, we computed it away from the thermodynamic limit for a single-qubit memory, showing it to be distinct from known free energies. We then described a proposal for approximating the optimal encoding strategy through a Jaynes-Cummings interaction of the memory with a Bosonic bath.

The connection between information processing and thermodynamics in general quantum settings has many facets. Among these, one that has stimulated significant interest is understanding the role of quantum effects such as coherence in thermodynamic contexts [16,38-47]. Our work provides a new perspective in investigating how quantum coherence improves our capacity to store information without additional free energy. Furthermore, passive information storage has operational relevance in sensing applications where the means through which a probe encodes environmental information is implicitly energy conserving $[29,30,32,33]$. The thermal information capacity then provides an ultimate upper bound in how much information such a probe can store.

Another natural question that follows from thermally passive encoding is. How can we use the encoded information in a way that is also subject to thermodynamic constraints? The primary challenge here is that the direct way to read out encoded information-measurement—lies outside the thermal operations framework, as measurementinduced collapse can drive systems out of thermal equilibrium. Hence, we need to explore more sophisticated frameworks, such as thermally passive coupling between the memory and the system it is storing information about. Developments in such directions could enable a full description of generalized quantum Szilard engines that take full advantage of initial memory states that can exist in quantum superpositions of nondegenerate energy eigenstates.

The authors thank Diane Donovan, Bevan Thompson, Syed Assad, and Ping Koy Lam for helpful discussions. The work is supported by the National Research Foundation of Singapore (NRF Fellowship Reference No. NRF-NRFF2016-02 and NRF Fellowship Reference No. NRF-CRP14-2014-02), the John Templeton Foundation (Grant No. 54914), the FQXi Large Grant "The role of quantum effects in simplifying adaptive agents," the National Research Foundation and L'Agence Nationale de la Recherche joint project NRF2017-NRF-
ANR004 VanQuTe, Huawei Technologies Grant "Quantum Algorithms and Models in Continuous and Discrete Variables", and the Singapore Ministry of Education Tier 1 RG190/17. G. G. acknowledges support from the Natural Sciences and Engineering Research Council of Canada.

*nvarun@ntu.edu.sg †mgu@quantumcomplexity.org

[1] L. Szilard, On the decrease of entropy in a thermodynamic system by the intervention of intelligent beings, Syst. Res. Behav. Sci. 9, 301 (1964).

[2] H.S. Leff and A. F. Rex, Maxwell's Demon: Entropy, Information, Computing (Princeton University Press, Princeton, NJ, 2014).

[3] R. Landauer, Irreversibility and heat generation in the computing process, IBM J. Res. Dev. 5, 183 (1961).

[4] C. H. Bennett, Notes on Landauer's principle, reversible computation, and Maxwell's demon, Stud. Hist. Philos. Sci. Part B 34, 501 (2003); Special issue: Quantum Information and Computation.

[5] T. Baumgratz, M. Cramer, and M. B. Plenio, Quantifying Coherence, Phys. Rev. Lett. 113, 140401 (2014).

[6] Z. Xi, Y. Li, and H. Fan, Quantum coherence and correlations in quantum system, Sci. Rep. 5, 10922 (2015).

[7] X. Yuan, H. Zhou, Z. Cao, and X. Ma, Intrinsic randomness as a measure of quantum coherence, Phys. Rev. A 92, 022124 (2015).

[8] A. Streltsov, U. Singh, H. Shekhar Dhar, M. Nath Bera, and G. Adesso, Measuring Quantum Coherence with Entanglement, Phys. Rev. Lett. 115, 020403 (2015).

[9] A. Winter and D. Yang, Operational Resource Theory of Coherence, Phys. Rev. Lett. 116, 120404 (2016).

[10] E. Chitambar and G. Gour, Critical Examination of Incoherent Operations and a Physically Consistent Resource Theory of Quantum Coherence, Phys. Rev. Lett. 117, 030401 (2016).

[11] E. Chitambar and M.-H. Hsieh, Relating the Resource Theories of Entanglement and Quantum Coherence, Phys. Rev. Lett. 117, 020402 (2016).

[12] J. Ma, B. Yadin, D. Girolami, V. Vedral, and M. Gu, Converting Coherence to Quantum Correlations, Phys. Rev. Lett. 116, 160407 (2016).

[13] Y. Peng, Y. Jiang, and H. Fan, Maximally coherent states and coherence-preserving operations, Phys. Rev. A 93, 032326 (2016).

[14] Y.-R. Zhang, L.-H. Shao, Y. Li, and H. Fan, Quantifying coherence in infinite-dimensional systems, Phys. Rev. A 93, 012334 (2016).

[15] M. O. Scully, M. Suhail Zubairy, G. S. Agarwal, and H. Walther, Extracting work from a single heat bath via vanishing quantum coherence, Science 299, 862 (2003).

[16] T. Sagawa and M. Ueda, Minimal Energy Cost for Thermodynamic Information Processing: Measurement and Information Erasure, Phys. Rev. Lett. 102, 250602 (2009).

[17] S. Wook Kim, T. Sagawa, S. De Liberato, and M. Ueda, Quantum Szilard Engine, Phys. Rev. Lett. 106, 070401 (2011). 
[18] O. C. O. Dahlsten, R. Renner, E. Rieper, and V. Vedral, Inadequacy of Von Neumann entropy for characterizing extractable work, New J. Phys. 13, 053015 (2011).

[19] F. G. S. L. Brandão, M. Horodecki, J. Oppenheim, J. M. Renes, and R. W. Spekkens, Resource Theory of Quantum States Out of Thermal Equilibrium, Phys. Rev. Lett. 111, 250404 (2013).

[20] M. Horodecki and J. Oppenheim, Fundamental limitations for quantum and nanoscale thermodynamics, Nat. Commun. 4, 2059 (2013).

[21] P. Skrzypczyk, A. J. Short, and S. Popescu, Work extraction and thermodynamics for individual quantum systems, Nat. Commun. 5, 4185 (2014).

[22] F. Binder, S. Vinjanampathy, K. Modi, and J. Goold, Quantum thermodynamics of general quantum processes, Phys. Rev. E 91, 032119 (2015).

[23] J. M. R. Parrondo, J. M. Horowitz, and T. Sagawa, Thermodynamics of information, Nat. Phys. 11, 131 (2015).

[24] F. Brandão, M. Horodecki, N. Ng, J. Oppenheim, and S. Wehner, The second laws of quantum thermodynamics, Proc. Natl. Acad. Sci. U.S.A. 112, 3275 (2015).

[25] G. Gour, M. P. Müller, V. Narasimhachar, R. W. Spekkens, and N. Yunger Halpern, The resource theory of informational nonequilibrium in thermodynamics, Phys. Rep. 583, 1 (2015).

[26] G. Gour, D. Jennings, F. Buscemi, R. Duan, and I. Marvian, Quantum majorization and a complete set of entropic conditions for quantum thermodynamics, arXiv:1708.04302.

[27] Equivalently, thermally passive encoding can be represented in terms of classical-quantum (CQ) states of the classical variable and the memory, whereby $\mathscr{C}(\rho)$ corresponds to the set of all CQ states accessible from $\rho$ under a generalized class of processes called conditioned thermal operations [28]. Since this more general framework is related but not essential to the present work, we shall state all of our results within the thermal operations framework.

[28] V. Narasimhachar and G. Gour, Resource theory under conditioned thermal operations, Phys. Rev. A 95, 012313 (2017).

[29] V. Giovannetti, S. Lloyd, and L. Maccone, Quantum Metrology, Phys. Rev. Lett. 96, 010401 (2006).

[30] V. Giovannetti, S. Lloyd, and L. Maccone, Advances in quantum metrology, Nat. Photonics 5, 222 (2011).

[31] S. Lloyd, Enhanced sensitivity of photodetection via quantum illumination, Science 321, 1463 (2008).

[32] S.-H. Tan, B. Erkmen, V. Giovannetti, S. Guha, S. Lloyd, L. Maccone, S. Pirandola, and J.H. Shapiro, Quantum Illumination with Gaussian States, Phys. Rev. Lett. 101, 253601 (2008).
[33] M. Bradshaw, S. Assad, J. Yan Haw, S.-H. Tan, P. Koy Lam, and M. Gu, Overarching framework between Gaussian quantum discord and Gaussian quantum illumination, Phys. Rev. A 95, 022333 (2017).

[34] The exact relationship between $F(\rho)$ and the Helmholtz free energy $A$ is given by $F(\rho)=\left[1 /\left(k_{\mathrm{B}} T\right)\right][A(\rho)-A(\gamma)]$, where $\gamma$ is the Gibbs state. $F(\rho)$ equals the maximum expected work extracted in a thermal process acting on initial state $\rho$, quantified in units of "work bits," i.e., single energy-degenerate qubit systems in pure states $[20,21,24]$.

[35] F. G. S. L. Brandão and G. Gour, Reversible Framework for Quantum Resource Theories, Phys. Rev. Lett. 115, 070503 (2015).

[36] See Supplemental Material at http://link.aps.org/ supplemental/10.1103/PhysRevLett.122.060601 for the thorough technical arguments.

[37] N. Yunger Halpern, Toward physical realizations of thermodynamic resource theories, in Information and Interaction (Springer, New York, 2017), pp. 135-166.

[38] M. Lostaglio, K. Korzekwa, D. Jennings, and T. Rudolph, Quantum Coherence, Time-Translation Symmetry, and Thermodynamics, Phys. Rev. X 5, 021001 (2015).

[39] P. Ćwikliński, M. Studziński, M. Horodecki, and J. Oppenheim, Limitations on the Evolution of Quantum Coherences: Towards Fully Quantum Second Laws of Thermodynamics, Phys. Rev. Lett. 115, 210403 (2015).

[40] V. Narasimhachar and G. Gour, Low-temperature thermodynamics with quantum coherence, Nat. Commun. 6, 7689 (2015).

[41] J. Åberg, Catalytic Coherence, Phys. Rev. Lett. 113, 150402 (2014).

[42] K. Korzekwa, M. Lostaglio, J. Oppenheim, and D. Jennings, The extraction of work from quantum coherence, arXiv: 1506.07875 .

[43] P. Kammerlander and J. Anders, Coherence and measurement in quantum thermodynamics, Sci. Rep. 6, 22174 (2016).

[44] L. Del Rio, J. Åberg, R. Renner, O. Dahlsten, and V. Vedral, The thermodynamic meaning of negative entropy, Nature (London) 474, 61 (2011).

[45] J. Jun Park, K.-H. Kim, T. Sagawa, and S. Wook Kim, Heat Engine Driven by Purely Quantum Information, Phys. Rev. Lett. 111, 230402 (2013).

[46] A. B. Boyd, D. Mandal, and J. P. Crutchfield, Identifying functional thermodynamics in autonomous Maxwellian ratchets, New J. Phys. 18, 023049 (2016).

[47] G. Francica, J. Goold, F. Plastina, and M. Paternostro, Daemonic ergotropy: Enhanced work extraction from quantum correlations., Quantum Inf. 3, 12 (2017). 